\title{
Atypical Functional Connectivity Associated with Autonomous Sensory Meridian Response: An Examination of Five Resting-State Networks
}

\author{
Stephen D. Smith, Beverley Katherine Fredborg, ${ }^{1,2}$ and Jennifer Kornelsen ${ }^{1,3}$
}

\begin{abstract}
Autonomous sensory meridian response (ASMR) is a perceptual phenomenon in which specific auditory and/or visual stimuli consistently elicit tingling sensations on the neck, scalp, and shoulders, as well as a positive and relaxed emotional state. The "ASMR triggers" that initiate these responses generally consist of soft sounds (e.g., whispering), repetitive noises (e.g., tapping sounds), or videos of people performing socially intimate acts (e.g., watching someone brush her hair). Despite being a relatively common phenomenon, little is known about the neural substrates of ASMR. In the current research, resting-state functional magnetic resonance imaging (fMRI) was used to examine whether ASMR was associated with atypical patterns of functional connectivity. Seventeen individuals with ASMR and 17 matched control participants underwent an anatomical MRI scan and a resting-state fMRI scan. An independent components analysis was used to identify the default mode, salience, central executive, sensorimotor, and visual networks. An analysis of variance with group (ASMR vs. control) as a between-subjects variable was performed to contrast the functional connectivity of each of these networks. The results demonstrated that ASMR was associated with reduced functional connectivity in the salience and visual networks, and with atypical patterns of connectivity in the default mode, central executive, and sensorimotor networks.
\end{abstract}

Keywords: autonomous sensory meridian response (ASMR); central executive network; default mode network; functional connectivity; resting-state networks

\section{Introduction}

A UTONOMOUS SENSORY MERIDIAN RESPONSE (ASMR) is a perceptual phenomenon in which specific auditory and visual stimuli reliably elicit tingling sensations in the head and neck, often extending down the back and limbs (Barratt and Davis, 2015). These somatosensory "tingles" are associated with a reduction in heart rate and an increase in skin conductance responses (Poerio et al., 2018). They are also typically accompanied by positive affect as well as a feeling of relaxation (Barratt and Davis, 2015). First reported in online forums in 2010, interest in ASMR has steadily increased with tens of thousands of ASMR-related videos on websites such as YouTube.com and subscribers to online discussion forums such as Reddit.com/r/ASMR exceeding 130,000. Despite this popularity and apparent prevalence, relatively few empirical studies have investigated ASMR. Therefore, little is known about the cognitive and neural mechanisms underlying these atypical sensory-emotional experiences. The goal of the current research was to identify patterns of neural activity that differentiate individuals with ASMR from the rest of the population.

To investigate an understudied phenomenon such as ASMR, it is important to identify the characteristics of the experience that make it distinct from other examples of atypical sensory associations such as synesthesia or frisson. Synesthesia refers to a "blending of the senses" in which the perception of one stimulus such as a letter or number (i.e., a "grapheme") automatically elicits a second percept such as the experience of seeing a color (i.e., a "photism"; Cytowic, 1993). Frisson, on the other hand, is described as the emotional "chills" associated with the perception of music or emotional events (Harrison and Loui, 2014).

\footnotetext{
${ }^{1}$ Department of Psychology, University of Winnipeg, Winnipeg, Canada.

${ }^{2}$ Department of Psychology, Ryerson University, Toronto, Canada.

${ }^{3}$ Department of Radiology, University of Manitoba, St. Boniface Hospital MRI Centre, Winnipeg, Canada.

(c) Stephen D. Smith et al., 2019; Published by Mary Ann Liebert, Inc. This Open Access article is distributed under the terms of the Creative Commons Attribution Noncommercial License (http://creativecommons.org/licenses/by-nc/4.0/) which permits any noncommercial use, distribution, and reproduction in any medium, provided the original author(s) and the source are cited.
} 
Although ASMR, synesthesia, and frisson are all examples of atypical multimodal experiences, some characteristics make ASMR distinct from these other phenomena (del Campo and Kehle, 2016). First, the stimuli that elicit ASMR-often referred to as "ASMR triggers"- - typically consist of socially intimate acts (e.g., watching someone apply makeup), whispering, and/or repetitive sounds (Barratt and Davis, 2015; Fredborg et al., 2017). This characteristic contrasts with synesthesia, which typically involves additional sensory responses to nonsocial stimuli such as graphemes, time-related concepts, and somatosensory experiences such as touching particular surfaces (Ward, 2013).

Second, the tingling sensations associated with ASMR are often described as dynamic and wave-like, rather than static and consistent (del Campo and Kehle, 2016). This characteristic is in stark contrast to synesthesia, where responses such as the photisms associated with grapheme-color synesthesia are usually identical across exposures and over time (Baron-Cohen et al., 1987; Cytowic, 1993).

Third, many individuals with ASMR are able to predict the types of stimuli that are most likely to elicit tingling sensations (Fredborg et al., 2017). Frisson responses, however, are not predictable; the same piece of music will not always produce "chills"; synesthetic responses are both automatic and predictable (Ramachandran and Hubbard, 2003; Ward, 2013). Taken together, these differences demonstrate that ASMR is a distinct perceptual and emotional phenomenon, likely relying on patterns of neural activity that differs from those recruited during other atypical sensory experiences.

There are two functional magnetic resonance imaging (fMRI) techniques that could be used to investigate the neural substrates of ASMR. In a task-based fMRI study, researchers could measure the brain activity during the ASMR response itself. This strategy is associated with some challenges. fMRI scanners are noisy environments; it is difficult to both relax and to hear soft, auditory ASMR triggers such as whispering in MRI scanners. That said, a recent study with 10 participants with ASMR was able to use fMRI to measure the neural activity while participants experienced tingles (Lochte et al., 2018). These researchers found that the experience of ASMR is associated with activity in the nucleus accumbens, dorsal anterior cingulate gyrus, insula, and inferior frontal gyrus. This activity in brain areas related to reward and emotional responses is consistent with the subjective reports of individuals experiencing ASMR (Barratt and Davis, 2015).

A second fMRI method is to examine how the brain activity of individuals with ASMR differs from those of matched control participants when no task is being performed. This strategy would help clarify why some individuals experience ASMR, whereas others do not. This differentiation could be accomplished by using resting-state fMRI, which measures fluctuations in the brain activity across different neural regions while the brain is "at rest" (i.e., not responding to a stimulus or performing a cognitive task). Previous research has demonstrated that the neural activity in disparate brain areas tends to fluctuate together (Biswal et al., 1995; Damoiseaux et al., 2006); this correlated activity suggests that these groups of neurons are working together as a network (Friston, 2011; Raichle, 2015). Numerous studies have found that the magnitude of the correlation between the activity of different groups of neurons-known as functional connectivity - can differentiate between different clinical and nonclinical populations
(Broyd et al., 2009; Greicius et al., 2004) and between individuals with high or low levels of different personality traits (Bilevicius et al., 2018; Doll et al., 2015).

To date, only one study has examined the functional connectivity in individuals with ASMR (Smith et al., 2017). This small study (with 11 ASMR and 11 control participants) focused exclusively on the functional connectivity of the default mode network (DMN), a task-negative network that has been linked to several cognitive functions including representations of the self (Andrews-Hanna, 2012), episodic memory (Sestieri et al., 2011), and mind-wandering (Gusnard and Raichle, 2001; Spreng et al., 2009). The DMN consists of the medial prefrontal cortex (mPFC), posterior inferior parietal lobule, angular gyrus, posterior cingulate cortex, and precuneus (Buckner et al., 2008; Raichle et al., 2001).

The functional connectivity of this network was found to be weaker in individuals with ASMR than in controls (Smith et al., 2017); however, the DMN of individuals with ASMR did appear to recruit additional brain regions, suggesting that the resting-state networks of ASMR participants were more blended (i.e., less distinct) than those of control participants. The purpose of the current study was to extend our previous research by examining the functional connectivity of additional resting-state networks, including the salience, central executive, sensorimotor, auditory, and visual networks; a larger sample size (17 ASMR and 17 control participants) will also allow us to more precisely assess the functional connectivity of the DMN in this population.

The salience network (SN) is involved with determining whether a sensory stimulus is salient in a given situation and whether attention and memory resources are required to react to the stimulus (Menon, 2015; Seeley et al., 2007). This network consists of the dorsal anterior cingulate cortex, the anterior insula, and the inferior frontal gyrus. Previous research has shown that the dorsal anterior cingulate cortex and anterior insula are sensitive to visceral feedback from the body (Craig, 2009; Critchley et al., 2004, 2005). Given that ASMR involves both visceral and emotional responses to a stimulus, an examination of this network is warranted.

The central executive network (CEN) is involved with attentional set shifting, response inhibition, working memory, and problem solving (Chen et al., 2016; Seeley et al., 2007), as well as with emotion regulation (Gagnepain et al., 2017). It includes the dorsolateral prefrontal cortex and posterior parietal cortex (Sridharan et al., 2008). In our previous investigation of ASMR, we speculated that individuals with ASMR may have a reduced ability to inhibit sensory-emotional responses that are suppressed in the rest of the population (Smith et al., 2017). Such a possibility may be related to altered CEN connectivity.

As ASMR is related to atypical associations between multiple sensory modalities, we also believed that it was important to examine the functional connectivity of different sensory networks. The sensorimotor network (SMN) consists of the primary motor cortex, premotor cortex, and supplementary cortex in both hemispheres (Rosazza and Minati, 2011). The visual network includes bilateral striate and extrastriate cortex; in some data sets, this network can be divided into medial and lateral components (Beckman et al., 2005). An effort was also made to investigate the auditory network, which includes the primary and secondary auditory cortices in the superior temporal lobe as well as the posterior insula (Beckmann et al., 2005; De Luca et al., 2006). Unfortunately, this network 
was not detected in our analyses. Therefore, the current investigation will examine whether the functional connectivity of five resting-state networks - the DMN, CEN, SN, SMN, and visual networks - differs between individuals with ASMR and matched control participants.

\section{Methods}

\section{Participants}

Seventeen participants (eight males) with ASMR between the ages of 18 and 37 years $\left(M_{\mathrm{age}}=22.71 ; S D_{\mathrm{age}}=4.74\right)$ were recruited from the Winnipeg, Manitoba community via wordof-mouth and social media posts. The presence of ASMR was confirmed by having participants view two popular videos that had been created to elicit ASMR responses. These videos were trimmed to be $6 \mathrm{~min}$ in length; the URLs for the full-length versions of these YouTube.com videos can be obtained by contacting the corresponding author. All ASMR participants confirmed that these videos elicited tingling sensations. This confirmation process was completed in the presence of one of the investigators, who further interviewed participants about their ASMR experiences to ensure that they experienced the response reliably.

Seventeen age-matched ( \pm 3 years) and sex-matched control participants were recruited from the University of Winnipeg student population. These participants also viewed the ASMR-eliciting videos in the presence of the investigators to confirm that the control participants did not experience ASMR. Data from two control participants were removed due to excessive movement during scanning; these individuals were replaced with two additional participants. The 17 control participants ( 8 males) included in the analyses had an age range of $18-40$ years $\left(M_{\text {age }}=22.76 ; S D_{\text {age }}=5.39\right)$.

Preliminary data from 22 of the 34 participants in the current study (11 ASMR and 11 control participants) were reported in a previous examination of the DMN (Smith et al., 2017). However, that small study did not report data related to any of the other four resting-state networks analyzed for the current research.

None of the participants had a history of psychiatric or neurological illness. This study was carried out in accordance with the recommendations of the Canadian TriCouncil Policy Statement: Ethical Conduct for Research Involving Humans (2014) and the Declaration of Helsinki, with written informed consent provided by all participants. The protocol was approved by the University of Manitoba's Bannatyne Human Research Ethics Board and the University of Winnipeg's Human Research Ethics Board. All participants completed MR safety screening before entering the MRI suite. Participants received \$50 CDN remuneration.

\section{Procedure}

Imaging began with a three-dimensional (3D), high-resolution anatomical MRI scan of the brain. This structural MRI took 8 min to complete. Following this, participants underwent a 7-min resting-state fMRI scan. During this scan, participants were instructed to remain still with their eyes closed.

\section{Data acquisition}

Data were acquired using a 3-Tesla Siemens TRIO MRI scanner (Siemens, Erlangen, Germany). For the anatom- ical scan, high-resolution, T1-weighted gradient-echo images were acquired using a magnetization-prepared rapidgradient-echo sequence with the following parameters: slice thickness $=1 \mathrm{~mm}$; interslice gap $=0 \mathrm{~mm}$; repetition time $(\mathrm{TR})=$ $1900 \mathrm{~ms}$; echo time $(\mathrm{TE})=2.2 \mathrm{~ms}$; in-plane resolution $=0.94 \times$ 0.94 ; matrix $=256 \times 256$; and field of view $(\mathrm{FOV})=24 \mathrm{~cm}$.

fMRI data acquisition consisted of a 7-min (140 volume) scan using a whole-brain echo-planar imaging sequence with the following parameters: slick thickness $=3 \mathrm{~mm}$; interslice gap $=0 \mathrm{~mm} ; \mathrm{TR}=3000 \mathrm{~ms} ; \mathrm{TE}=30 \mathrm{~ms}$; flip angle $=90^{\circ}$; matrix $=64 \times 64$; and $\mathrm{FOV}=24 \mathrm{~cm}$.

\section{Data analysis}

Imaging data were processed using BrainVoyager QX 3.6 software (Brain Innovation, Maastricht, The Netherlands). Functional data underwent four preprocessing procedures. A trilinear/sync interpolation 3D motion correction procedure provided an estimate of each participant's movement in three translations and three rotations, which were then regressed out of the data before the individual-level analyses were performed. The functional data also underwent a slice scan time correction and high-pass temporal filtering. Spatial smoothing consisted of an $8 \mathrm{~mm}$ full-width half-maximum Gaussian filter (Mikl et al., 2008). Following preprocessing, each participant's functional data were co-registered to his or her anatomical MRI. Each participant's anatomical scan was manually transformed to Talairach space, and the coregistration parameters were used to warp the functional scan data.

Each participant's resting-state data were subjected to a single-subject independent components analysis (ICA) using a fast ICA algorithm (Hyvärinen and Oja, 2000). Twenty individual components were extracted for each participant's data (Abou-Elseoud et al., 2010; Ray et al., 2013). A self-organizing group-level ICA (Esposito et al., 2005) was then performed using the data from all 34 participants. The components from this analysis were then inspected to identify the DMN, SN, CEN, SMN, auditory, and visual resting-state networks. Unfortunately, the auditory network did not clearly load onto any of the components, thus preventing further analyses of this network. The coordinates of the remaining five networks were compared with the Talairach coordinates identified in previously published experiments.

A two-factor, mixed-effects analysis of variance (ANOVA) was then performed, with the within-subjects factor being the 20 fixed levels ( 20 components) and the between-subjects factor being the two groups (ASMR and control participants). Specific contrasts were performed for each of the five identified networks $(t=2.58, p<0.01$, uncorrected) and corrected for multiple comparisons with a cluster threshold estimator plugin using Monte Carlo simulations at 1000 iterations.

Each contrast map was converted to volumes of interest to determine the number and coordinates of voxels exceeding the statistical threshold. This process also identified the Talairach coordinates of the peak activation point as well as the probability value associated with each cluster. The peak activity coordinates were entered into Talairach Client software; this program provided the anatomical names and Brodmann areas associated with each coordinate.

To gain a more complete understanding of the extent and characteristics of each cluster, a list of coordinates of each 
significant voxel in each cluster was obtained. These lists were then entered into Talairach Client software to identify all the brain structures comprising each cluster.

Additional post hoc analyses were performed. Upon inspection of the remaining 15 components, 1 additional component appeared to include both the DMN and CEN, anticorrelated. We ran this sixth component through the identical analysis procedure outlined above for the five identified networks. In addition, post hoc analyses using age and sex as covariates of the ASMR versus control comparisons were also performed. These variables did not affect the current results and will not be discussed below; however, it is important to note that our sample size may not have been sufficient to detect these effects.

\section{Results}

The DMN, SN, CEN, SMN, and visual network were all detected as single components in the group-level ICA. Of note, although the CEN is often detected as two separate components-left CEN and right $\mathrm{CEN}$ - in the current study, one component included CEN connectivity in both hemispheres. Images of these five networks are shown in Figure 1. Separate ANOVAs were performed to identify between-group differences in each of these five networks.

In the examination of the DMN, the functional connectivity of two brain structures differed between ASMR and control participants (Table 1). Control participants showed greater connectivity than ASMR participants in the left precuneus, one of the key nodes of the DMN. This cluster was relatively large, encompassing 7379 voxels. In contrast, the DMN of individuals with ASMR appeared to recruit a smaller (1834 voxel) cluster in the cuneus, a visual region that is adjacent to the precuneus component of the DMN.

The analysis of the SN also showed intergroup differences; however, in this case, all the differences consisted of greater functional connectivity in controls relative to ASMR participants (Table 2). In the left hemisphere, a significant difference was detected in the inferior frontal gyrus. This cluster extended into the left insula as well as into the left claustrum and putamen. A second lefthemisphere cluster showed peak activation in the superior temporal gyrus (Brodmann's area [BA] 22). This cluster also included the left insula as well as the inferior parietal lobule and several subregions of the posterior middle (BA 21, 22, and 39) and superior (BA 13, 22, 39, and 42) temporal gyri. Two significant differences were identified in the right hemisphere as well. One region had peak activation in the right superior temporal gyrus (BA 46). This cluster extended into the right insula, a brain area linked with interoception (Craig, 2009), the postcentral gyrus, and three subregions of the posterior superior temporal gyrus (BA 21, 41, and 42). The final cluster was located in visual regions of the right hemisphere including the middle occipital gyrus, lingual gyrus, and cuneus. Taken together, the results of the analysis of the SN suggest that this network show stronger, more coherent functional connectivity in control participants than in individuals with ASMR. It is also noteworthy that two of these clusters were in brain areas involved with audition, suggesting that the link between auditory perception and salience judgments may be weaker in individuals with ASMR.

Six intergroup differences were identified in the ANOVA examining the CEN functional connectivity (Table 3). Of these, five clusters indicated greater functional connectivity in control participants, and one was associated with increased connectivity in ASMR participants. In the left hemisphere, two large clusters indicated greater functional connectivity in control participants. One of these clusters included voxels in the middle and superior frontal gyri. Peak intensity in the second cluster was in the posterior cingulate gyrus but included voxels in the cuneus, precuneus, middle occipital gyrus, lingual gyrus, parahippocampal gyrus, insula, supramarginal gyrus, and middle and superior temporal gyri. In the right hemisphere, control participants showed greater functional connectivity than ASMR participants in three areas. These clusters resembled the connectivity detected in the left hemisphere. One cluster was isolated to the right middle and superior frontal gyri. A second cluster included the middle occipital gyrus, cuneus, and posterior cingulate gyrus. The third cluster was detected in the right insula, extending into the middle and superior gyri. When combined, these latter two clusters resemble the large left hemisphere cluster that included several occipital and temporal lobe structures. It is possible that at a less conservative statistical threshold, these clusters would have appeared as a single large cluster as they did in the left hemisphere. Finally, greater functional connectivity was detected in ASMR participants relative to control participants in the middle and posterior sections of the left cingulate gyrus (BA 23 and 24). Together, these patterns of connectivity show increased bilateral connectivity in frontal and occipitotemporal regions in control participants. ASMR, however, was associated with increased connectivity in the midcingulate cortex.

Six intergroup differences were also identified in the ANOVA examining the functional connectivity in the SMN (Table 4). Three of these differences indicated greater functional connectivity in control participants. One of these clusters included the anterior cingulate gyrus (BA 24 and 32), the precentral cortex, and premotor regions (BA 6). A second cluster not only included the bilateral connectivity in the posterior cingulate gyrus and precuneus but also included the left paracentral lobule, a region involved with sensation and movement. The third cluster associated with greater functional connectivity in controls included the body and head of the caudate in both hemispheres, as well as the left globus pallidus, putamen, and subgenual anterior cingulate gyrus (BA 25). There were also three intergroup differences reflecting greater functional connectivity in the brains of ASMR participants. One cluster was isolated in the left precentral and postcentral gyri. As all participants were right-handed, it is unclear whether this lateralization is related to handedness. A second cluster had its peak activity in the left parahippocampal gyrus but extended into the left amygdala, hippocampus, and uncus. The third cluster, which showed greater connectivity in the ASMR group, was also the only cluster that was right lateralized and included the right orbital, middle frontal, and superior frontal gyri. These results suggest that control participants had greater functional connectivity in many motoric areas, whereas individuals with ASMR showed increased connectivity in the primary somatosensory cortex as well as regions related to emotion and reward responses (i.e., the amygdala and orbital gyrus). 


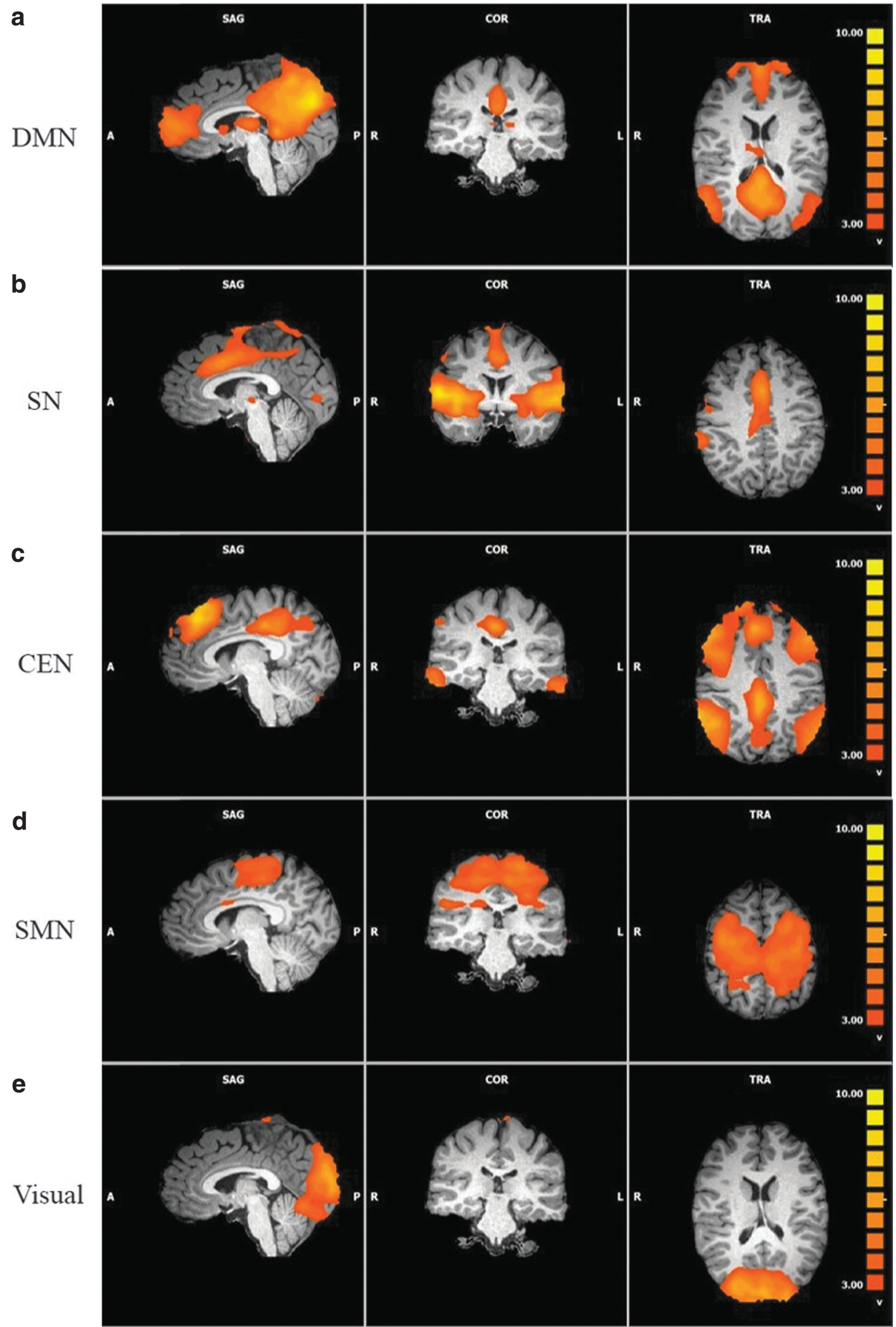

FIG. 1. Resting-state networks of 34 participants. Functional connectivity of the (a) DMN, (b) SN, (c) CEN, (d) SMN, and (e) visual network identified in the group-level ICA. The data from all 34 participants are included in these data. CEN, central executive network; DMN, default mode network; ICA, independent components analysis; SMN, sensorimotor network; SN, salience network. 
Table 1. Contrasting the Functional Connectivity of Brain Regions in the Default Mode Network of Autonomous Sensory Meridian Response Participants and Matched Control Participants

\begin{tabular}{|c|c|c|c|c|c|c|c|c|c|}
\hline \multirow[b]{2}{*}{ Hemisphere } & \multirow[b]{2}{*}{ Region } & \multirow[b]{2}{*}{ Structure } & \multicolumn{7}{|c|}{ Talairach coordinates } \\
\hline & & & $B A$ & $\mathrm{X}$ & $\mathrm{Y}$ & $\mathrm{Z}$ & Cluster size & $\mathrm{t}$ & $\mathrm{p}$ \\
\hline \multicolumn{10}{|c|}{ Controls $>$ ASMR } \\
\hline Left & Parietal & Precuneus & 7 & -7 & -62 & 39 & 7379 & 4.2952 & 0.00002 \\
\hline \multicolumn{10}{|c|}{ ASMR > controls } \\
\hline Left & Occipital & Cuneus & 18 & -7 & -83 & 21 & 1834 & -3.6063 & 0.00033 \\
\hline
\end{tabular}

The Talairach coordinates of the peak intensity voxel of each cluster is listed, along with the associated brain region and BA.

ASMR, autonomous sensory meridian response; BA, Brodmann's area.

The final resting-state network examined in our initial analyses was the visual network. As with the SN, all intergroup differences indicated greater functional connectivity in the brains of control participants (Table 5). Two relatively symmetrical differences were observed in the left and right lingual gyri. Both clusters also included voxels in the parahippocampal and posterior cingulate gyri and extended into the culmen, declive, tuber, and pyramis of the cerebellum. The rightlateralized cluster contained additional voxels in the fusiform gyrus and cuneus. A third cluster consisted of the right superior temporal gyrus, precentral gyrus, and insula, regions related to movement and interoception. A final cluster was isolated to right cerebellar structures, with the majority of the voxels located in the declive and uvula, regions related to oculomotor functions (Ruehl et al., 2017). The results of this ANOVA suggest that control participants have more robust functional connectivity within traditional nodes of the visual network and show stronger connectivity between this network and regions related to movement.

\section{Post hoc analyses of a "blended" network}

The initial analyses of the DMN, SN, CEN, SMN, and visual network suggested that, overall, the functional connectivity of individuals with ASMR was much less coherent than that of control participants. However, a post hoc inspection of the 15 remaining components from our ICA analysis revealed 1 component that appeared to contain anticorrelated elements of a DMN and a CEN (Fig. 2); there was no discernable pattern to the connectivity of the remaining 14 components. An additional ANOVA was therefore performed on this "blended" component. The results indicated that there were 11 significant differences between the ASMR and control participants, 5 in- dicating greater functional connectivity in controls and 6 indicating greater functional connectivity in individuals with ASMR $(t=2.58, p<0.01$, corrected for multiple comparisons using a cluster threshold estimator; Table 6).

All five of the clusters depicting greater functional connectivity in control participants were lateralized to the right hemisphere. Two of these clusters were located in the frontal lobes. One cluster consisted of voxels in inferior and middle frontal gyri, extending into the right precentral gyrus. The second frontal lobe cluster consisted of voxels in the middle and superior frontal gyri. A third cluster had its peak connectivity in the temporal lobe, with voxels in the inferior and middle temporal gyri. The remaining two clusters were located in the right parietal lobe. One cluster included cells in the inferior parietal lobule and supramarginal gyrus, whereas the other cluster consisted of voxels in the precuneus and superior parietal lobule. This pattern of connectivity shares some characteristics with both a right-lateralized CEN and the right-hemisphere portion of the DMN, although a bilateral CEN and DMN were clearly detected in different components of the ICA.

The six clusters depicting greater functional connectivity in ASMR participants are notable in that four clusters are in regions that showed greater connectivity in control participants in the CEN component. Clusters in the left and right superior frontal gyri, the left middle frontal gyrus, and the left posterior cingulate gyrus all overlapped with clusters detected in the CEN ANOVA (Table 3). ASMR was also associated with greater functional connectivity in a left temporoparietal area including the middle and superior temporal gyri, the inferior and superior parietal lobules, and the angular and supramarginal gyri. This large cluster appears to mirror three of the right-lateralized clusters showing greater

Table 2. Contrasting the Functional Connectivity of Brain Regions in the Salience Network of Autonomous Sensory Meridian Response Participants and Matched Control Participants

\begin{tabular}{|c|c|c|c|c|c|c|c|c|c|}
\hline \multirow[b]{2}{*}{ Hemisphere } & \multirow[b]{2}{*}{ Region } & \multirow[b]{2}{*}{ Structure } & \multicolumn{7}{|c|}{ Talairach coordinates } \\
\hline & & & $B A$ & $\mathrm{X}$ & Y & $\mathrm{Z}$ & Cluster size & $\mathrm{t}$ & $\mathrm{p}$ \\
\hline \multicolumn{10}{|c|}{ Controls $>$ ASMR } \\
\hline Left & Frontal & Inferior frontal gyrus & 46 & -34 & 31 & 9 & 2606 & 4.0840 & 0.00005 \\
\hline Left & Temporal & Superior temporal gyrus & 22 & -65 & -35 & 9 & 7200 & 4.5752 & 0.00001 \\
\hline Right & Temporal & Superior temporal gyrus & 42 & 59 & -29 & 12 & 3598 & 4.2524 & 0.00002 \\
\hline Right & Occipital & Middle occipital gyrus & 18 & 26 & -83 & -3 & 1696 & 3.5265 & 0.00045 \\
\hline \multicolumn{10}{|c|}{ ASMR > controls } \\
\hline
\end{tabular}

The Talairach coordinates of the peak intensity voxel of each cluster is listed, along with the associated brain region and BA. 
Table 3. Contrasting the Functional Connectivity of Brain Regions in the Central Executive Network of Autonomous Sensory Meridian Response Participants and Matched Control Participants

\begin{tabular}{|c|c|c|c|c|c|c|c|c|c|}
\hline \multirow[b]{2}{*}{ Hemisphere } & \multirow[b]{2}{*}{ Region } & \multirow[b]{2}{*}{ Structure } & \multicolumn{7}{|c|}{ Talairach coordinates } \\
\hline & & & $B A$ & $\mathrm{X}$ & Y & $\mathrm{Z}$ & Cluster size & $\mathrm{t}$ & $\mathrm{p}$ \\
\hline \multicolumn{10}{|c|}{ Controls $>$ ASMR } \\
\hline Left & Limbic & Posterior cingulate gyrus & 30 & -28 & -65 & 12 & 18166 & 4.2837 & 0.00002 \\
\hline Left & Frontal & Middle frontal gyrus & 10 & -22 & 67 & 22 & 14563 & 5.3659 & 0.00000 \\
\hline Right & Frontal & Superior frontal gyrus & 10 & 17 & 64 & 30 & 2175 & 4.6678 & 0.00000 \\
\hline Right & Occipital & Middle occipital gyrus & 18 & 26 & -80 & 9 & 5462 & 3.9924 & 0.00007 \\
\hline Right & Sublobar & Insula & 13 & 41 & -44 & 15 & 3718 & 3.9094 & 0.00010 \\
\hline \multicolumn{10}{|c|}{ ASMR >controls } \\
\hline Left & Limbic & Cingulate gyrus & 24 & -10 & -17 & 33 & 6495 & -4.1553 & 0.00004 \\
\hline
\end{tabular}

The Talairach coordinates of the peak intensity voxel of each cluster is listed, along with the associated brain region and BA.

functional connectivity in control participants. An additional cluster included voxels in the right middle and superior temporal gyri, extending into the angular gyrus.

The fact that individuals with ASMR showed greater functional connectivity in some brain areas associated with the CEN in a component that was distinct from the previously analyzed bilateral CEN component suggested that further investigation was required. In our previous study, we found that ASMR was associated with a less distinct DMN (Smith et al., 2017). It is possible that additional restingstate networks are also less coherent in this population. To examine this possibility, we conducted a post hoc grouplevel ICA using only the data from the 17 ASMR participants. Consistent with our hypothesis, we found that the DMN of ASMR participants appeared as separate anterior and posterior components. Similarly, the CEN appeared as separate left and right components. Thus, the differences detected in the intergroup ANOVAs were likely due to the fact that the resting-state networks of ASMR participants were more fractionated than those of control participants.

To control for this fractionation, we conducted one additional post hoc ANOVA in which the group-level ICA components associated with the DMN, the CEN, and the additional blended component were combined. When the data from these combined components were compared across the ASMR and control groups, we found two significant differences between the two groups (Fig. 3 and Table 7). Control participants showed greater functional connectivity than
ASMR participants in a cluster consisting of the precuneus and superior parietal lobule of both hemispheres; a larger number of significant voxels were detected in the right than in the left hemisphere. In contrast, greater functional connectivity was detected in the brains of ASMR participants in a large midline cluster consisting of the left and right cingulate gyri (BA 23 and 24), and left precentral, inferior frontal, and middle frontal gyri. Thus, even when combining ICA components to account for the possible fractionation of the DMN and CEN, there are still two important differences: control participants show relatively greater functional connectivity in a key node of the DMN and ASMR participants show greater connectivity in regions related to movement and salience detection.

\section{Discussion}

The data from the current study suggest that the restingstate networks of individuals with ASMR are less distinct than those of controls. Of the 22 clusters that differed between ASMR and control participants, 17 showed greater functional connectivity in controls. The reduced functional connectivity found across resting-state networks in ASMR is consistent with our earlier study of the DMN (Smith et al., 2017). In that study, several nodes of the DMN including the precuneus and mPFC showed less connectivity in participants with ASMR than in controls. It is also consistent with resting-state investigations of synesthesia; Dovern

Table 4. Contrasting the Functional Connectivity of Brain Regions in the Sensorimotor Network of Autonomous Sensory Meridian Response Participants and Matched Control Participants

\begin{tabular}{|c|c|c|c|c|c|c|c|c|c|}
\hline \multirow[b]{2}{*}{ Hemisphere } & \multirow[b]{2}{*}{ Region } & \multirow[b]{2}{*}{ Structure } & \multicolumn{7}{|c|}{ Talairach coordinates } \\
\hline & & & $B A$ & $\mathrm{X}$ & Y & $\mathrm{Z}$ & Cluster size & $\mathrm{t}$ & $\mathrm{p}$ \\
\hline \multicolumn{10}{|c|}{ Controls $>$ ASMR } \\
\hline Left & Limbic & Cingulate gyrus & 24 & -19 & 1 & 42 & 2326 & 3.4744 & 0.00055 \\
\hline Left & Limbic & Posterior cingulate gyrus & 31 & -19 & -38 & 42 & 5000 & 4.3216 & 0.00002 \\
\hline Left & Sub-lobar & Caudate & $*$ & -10 & 7 & -6 & 2601 & 3.8245 & 0.00014 \\
\hline \multicolumn{10}{|c|}{ ASMR $>$ controls } \\
\hline Left & Frontal & Precentral gyrus & 4 & -55 & -17 & 36 & 2099 & -3.9222 & 0.00010 \\
\hline Left & Limbic & Parahippocampal gyrus & 34 & -16 & -8 & -21 & 2058 & -4.8400 & 0.00000 \\
\hline Right & Frontal & Orbital gyrus & 11 & 8 & 43 & -19 & 1539 & -3.4934 & 0.00051 \\
\hline
\end{tabular}

The Talairach coordinates of the peak intensity voxel of each cluster is listed, along with the associated brain region and BA. 
Table 5. Contrasting the Functional Connectivity of Brain Regions in the Visual Network of Autonomous Sensory Meridian Response Participants and Matched Control Participants

\begin{tabular}{|c|c|c|c|c|c|c|c|c|c|}
\hline \multirow[b]{2}{*}{ Hemisphere } & \multirow[b]{2}{*}{ Region } & \multirow[b]{2}{*}{ Structure } & \multicolumn{7}{|c|}{ Talairach coordinates } \\
\hline & & & $B A$ & $X$ & $\mathrm{Y}$ & $\mathrm{Z}$ & Cluster size & $\mathrm{t}$ & $\mathrm{p}$ \\
\hline \multicolumn{10}{|c|}{ Controls $>$ ASMR } \\
\hline Left & Occipital & Lingual gyrus & 19 & -25 & -59 & 0 & 7197 & 4.2119 & 0.00003 \\
\hline Right & Temporal & Superior temporal gyrus & 22 & 62 & -11 & 6 & 6168 & 3.9379 & 0.00009 \\
\hline Right & Occipital & Lingual gyrus & 18 & 11 & -56 & 6 & 8192 & 4.0608 & 0.00006 \\
\hline Right & Cerebellum & Declive & $*$ & 14 & -74 & -18 & 5249 & 5.3789 & 0.00000 \\
\hline \multicolumn{10}{|c|}{ ASMR $>$ controls } \\
\hline
\end{tabular}

The Talairach coordinates of the peak intensity voxel of each cluster is listed, along with the associated brain region and BA.

and colleagues (2012) found that nonsynesthetes displayed greater functional connectivity in 11 clusters spread across 7 networks, whereas synesthetes showed greater connectivity in 3 networks. However, a study of aesthetic chill, a phenomenon similar to frisson (Benedek and Kaernbach, 2011; McCrae, 2007) reported that this experience was associated with greater connectivity between the DMN and a number of regions including the sensory and motor cortices and the SN (Williams et al., 2018). Although the data from different phenomenon are not entirely consistent, these studies do suggest that atypical multimodal experiences are linked with altered patterns of functional connectivity, particularly involving the DMN.

The post hoc analyses conducted for the current study suggest that the DMN, and its relationship to other networks, differs between ASMR and control participants. The ICA of the ASMR group alone revealed that the anterior and posterior DMN and the left and right CEN were each represented in independent components, suggesting the connectivity among the key nodes of these networks are perhaps less robust in ASMR compared with controls. This may have contributed to the formation of the additional blended ICA component in the initial grouped analysis.

An additional difference between ASMR and control participants in the current study was the functional connectivity of superior temporal lobe regions typically associated with auditory perception and the processing of vocal information (Hickok and Poeppel, 2007). In control participants, the activ- ity in the left and right superior temporal gyri fluctuated in synchrony with the SN (Table 2). In contrast, ASMR participants showed synchronous activity between structures related to the CEN and the posterior and middle temporal lobesregions related to the processing of speech - in the blended ICA component (Table 6). This latter finding is particularly intriguing given the sensitivity of many individuals with ASMR to whispering (Barratt and Davis, 2015; Fredborg et al., 2017). The potential role of superior temporal lobe structures in ASMR could be assessed in future task-based fMRI studies examining whether this region shows differential sensitivity to vocal and nonvocal ASMR triggers.

Two examples of atypical connectivity are directly related to the sensory-emotional nature of ASMR phenomenology. The first involves the functional connectivity of the SMN. In individuals with ASMR, the activity of the SMN also correlated with the activity of a cluster that included the orbital gyrus. This brain area is part of the frontal lobe reward valuation network that responds to both sensory and social rewards (Grabenhorst and Rolls, 2011); these rewards can be personal or vicarious in nature (Morelli et al., 2015), an important point given that many ASMR triggers involve a third-person perspective (Fredborg et al., 2017). The fact that the activity of neurons in reward-sensitive regions correlate highly with the SMN suggests a stronger link between these two functions in individuals with ASMR and may help explain why these individuals experience rewarding tingling sensations.
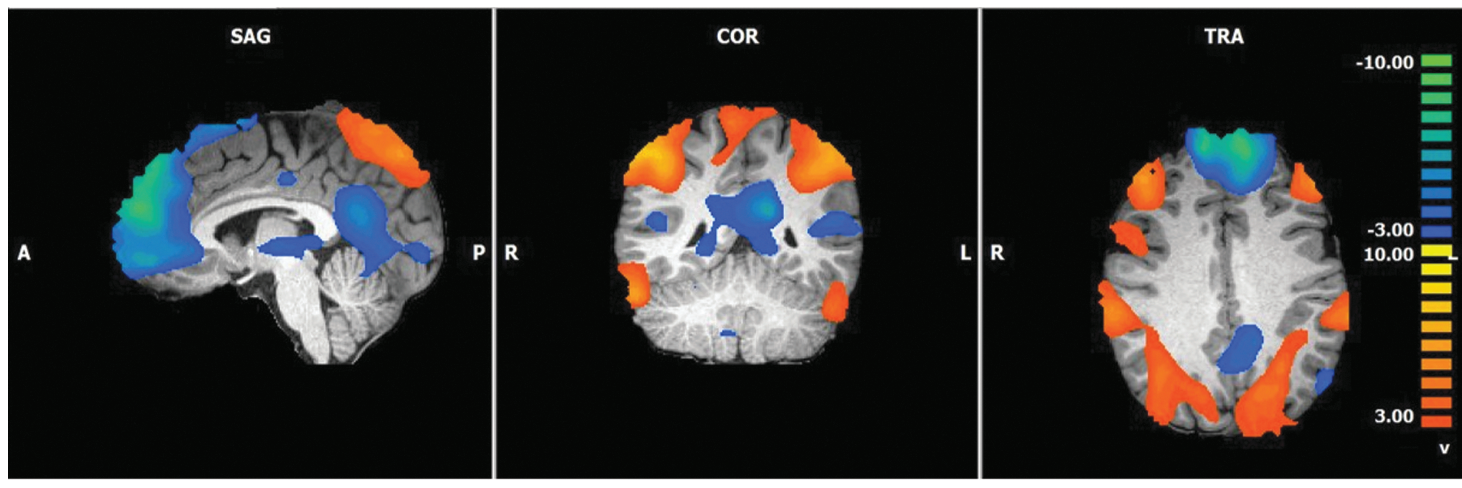

FIG. 2. Functional connectivity of a "blended network." Functional connectivity in the blended network of individuals with ASMR and matched controls. This network contains elements of an anterior DMN and a CEN. The orange and blue voxels do not differentiate between ASMR and control participants in this figure; rather, they depict regions whose functional connectivity is anticorrelated. ASMR, autonomous sensory meridian response. 
Table 6. Post hoc Contrast of the Functional Connectivity of Brain Regions in the "Blended Network" of Autonomous Sensory Meridian Response Participants AND Matched CONTROL Participants

\begin{tabular}{|c|c|c|c|c|c|c|c|c|c|}
\hline \multirow[b]{2}{*}{ Hemisphere } & \multirow[b]{2}{*}{ Region } & \multirow[b]{2}{*}{ Structure } & \multicolumn{7}{|c|}{ Talairach coordinates } \\
\hline & & & $B A$ & $X$ & $\mathrm{Y}$ & $\mathrm{Z}$ & Cluster size & $\mathrm{t}$ & $\mathrm{p}$ \\
\hline \multicolumn{10}{|c|}{ Controls $>$ ASMR } \\
\hline Right & Frontal & Superior frontal gyrus & 6 & 17 & -2 & 69 & 2646 & 4.6440 & 0.00000 \\
\hline Right & Frontal & Inferior frontal gyrus & 47 & 44 & 31 & -3 & 9157 & 4.0252 & 0.00006 \\
\hline Right & Temporal & Inferior temporal gyrus & 20 & 50 & -32 & -12 & 4014 & 4.1487 & 0.00004 \\
\hline Right & Parietal & Inferior parietal lobule & 40 & 44 & -44 & 39 & 1816 & 4.1880 & 0.00003 \\
\hline Right & Parietal & Precuneus & 7 & 23 & -71 & 48 & 3042 & 3.8515 & 0.00013 \\
\hline \multicolumn{10}{|c|}{ ASMR $>$ controls } \\
\hline Left & Frontal & Superior frontal gyrus & 6 & -13 & 34 & 57 & 2655 & -4.2801 & 0.00002 \\
\hline Left & Frontal & Middle frontal gyrus & 6 & -46 & 7 & 48 & 5667 & -3.5623 & 0.00040 \\
\hline Left & Temporal & Superior temporal gyrus & 39 & -40 & -56 & 21 & 9881 & -5.3777 & 0.00000 \\
\hline Left & Limbic & Posterior cingulate gyrus & 31 & -4 & -47 & 27 & 3302 & -4.1859 & 0.00003 \\
\hline Right & Frontal & Superior frontal gyrus & 10 & 2 & 68 & 21 & 3305 & -4.5738 & 0.00001 \\
\hline Right & Temporal & Middle temporal gyrus & 39 & 50 & -62 & 24 & 2623 & 4.1467 & 0.00004 \\
\hline
\end{tabular}

The Talairach coordinates of the peak intensity voxel of each cluster is listed, along with the associated brain region and BA.

A second intriguing pattern relates to the functional connectivity of the midcingulate cortex (BA 24). This region showed greater functional connectivity in the CEN in ASMR participants than in controls. ASMR participants also showed increased functional connectivity in this region in the ANOVA combining the DMN, CEN, and blended components; the peak activation point of this cluster was the primary motor cortex. Previous research has shown that the midcingu- late cortex serves as an interaction point between emotional and motoric responses (Pereira et al., 2010). It is possible that ASMR is associated with an increased emotional modulation of motoric functioning.

Given that this article was the first to examine multiple resting-state networks in the ASMR population, it was logical to use a technique that examined the entire brain. This "hypothesis free" ICA approach detected structures, such
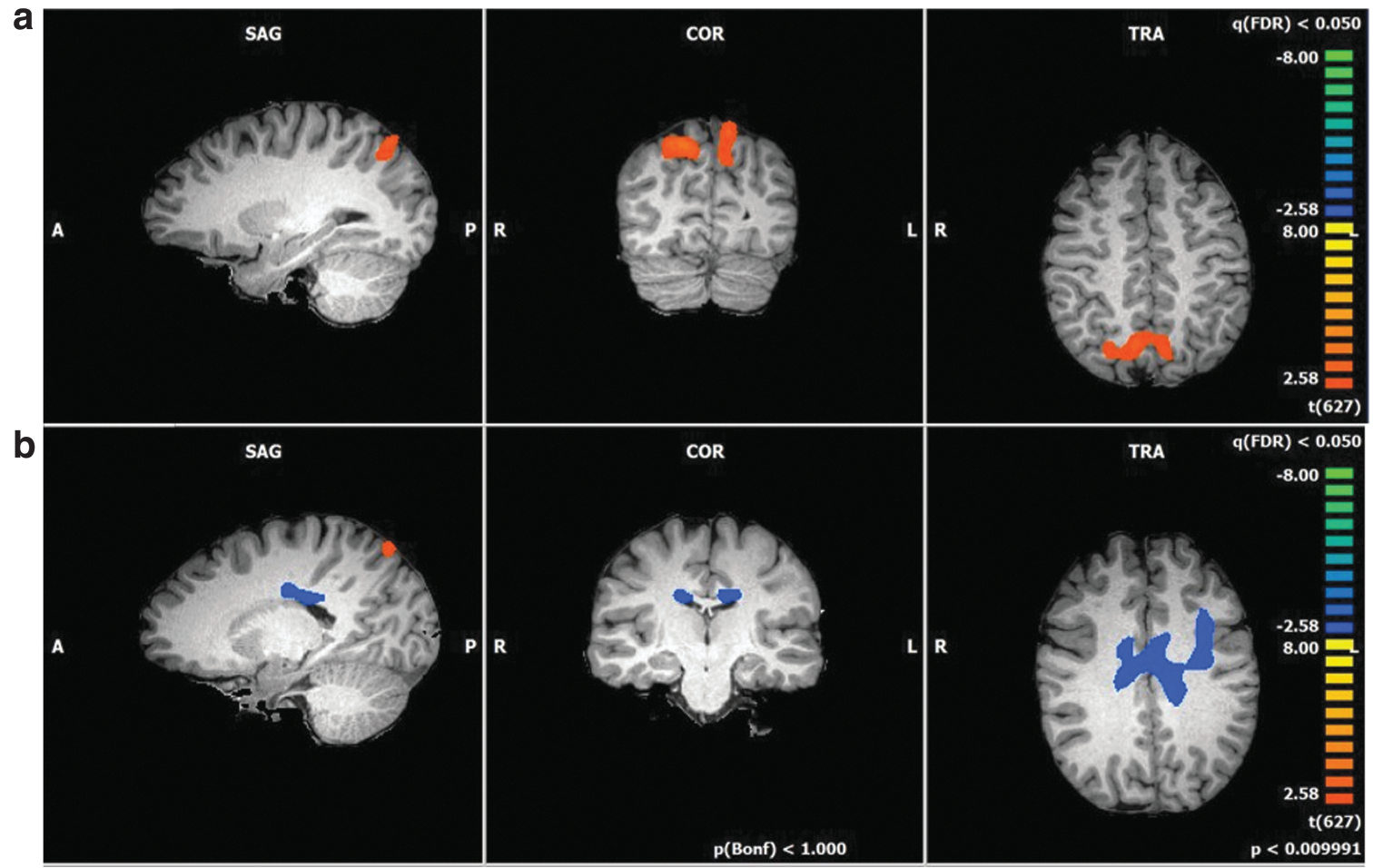

FIG. 3. Combined analysis of the DMN, CEN, and blended network. A comparison of functional connectivity occurring in a combined analysis of the DMN, CEN, and blended component (post hoc analysis) of control participants and individuals with ASMR $(t=2.58, p<0.01$; corrected for multiple comparisons via Monte Carlo simulations at 1000 iterations). (a) Orange voxels indicate regions in which greater functional connectivity was observed in control participants. (b) Blue voxels indicate regions in which greater functional connectivity was observed in ASMR participants. 
Table 7. Post hoc Contrast of the Functional Connectivity of Autonomous Sensory Meridian Response Participants and Matched Control Participants When the Default Mode Network, Central Executive Network, and the Blended Network Are Combined

\begin{tabular}{|c|c|c|c|c|c|c|c|c|c|}
\hline \multirow[b]{2}{*}{ Hemisphere } & \multirow[b]{2}{*}{ Region } & \multirow[b]{2}{*}{ Structure } & \multicolumn{7}{|c|}{ Talairach coordinates } \\
\hline & & & $B A$ & $X$ & Y & $\mathrm{Z}$ & Cluster size & $\mathrm{t}$ & $\mathrm{p}$ \\
\hline \multicolumn{10}{|c|}{ Controls $>$ ASMR } \\
\hline Right & Parietal & Precuneus & 7 & 14 & -74 & 51 & 6797 & 4.1661 & 0.00004 \\
\hline \multicolumn{10}{|c|}{ ASMR > controls } \\
\hline Left & Frontal & Precentral gyrus & 6 & -34 & 4 & 24 & 8649 & -3.4319 & 0.00064 \\
\hline
\end{tabular}

The Talairach coordinates of the peak intensity voxel of each cluster is listed, along with the associated brain region and BA.

as the orbital gyrus and midcingulate gyrus, which likely would not have been included in seed-based examinations of the functional connectivity. However, the ICA approach did not allow us to quantify the relationship between networks or to trace the functional connectivity of specific structures that are relevant to the phenomenology of ASMR, such as the right insula or somatosensory cortices. These questions must be addressed in the future research. Importantly, these seed-based functional connectivity studies can potentially use coordinates from the current research to provide a more complete description of the connectivity of different ASMRrelated brain structures (Joel et al., 2011). A similar strategy could be used for investigations of structural connectivity differences between ASMR and control participants.

\section{Limitations}

Although the current study provides novel information about the neural underpinnings of ASMR, it does have a number of limitations. The sample size (17 ASMR and 17 control participants) was relatively modest, and the thresholds used were somewhat liberal $(p<0.01)$. Additionally, the data do not take into account the fact that the intensity of ASMR varies across individuals (Fredborg et al., 2017), a factor that may have influenced the observed patterns of data. Future studies should therefore include a test of participants' sensitivity to different ASMR triggers as well as a larger sample size. A more conservative threshold of $p<0.001$ would also further decrease the likelihood of detecting "false positives" (Woo et al., 2014).

\section{Conclusion}

The current study demonstrates that the functional connectivity of individuals with ASMR differs from that of control participants. The weaker connectivity detected in numerous networks suggests that the resting-state networks of individuals with ASMR are not as distinct as in the brains of non-ASMR individuals. Additionally, the recruitment of orbitofrontal cortex neurons by the SMN of ASMR participants may indicate one of the neural substrates underlying the rewarding tingling sensations associated with ASMR.

\section{Acknowledgments}

This research was funded by the Natural Sciences and Engineering Research Council (NSERC) of Canada. The authors wish to thank Teresa Figley and the radiology technical staff for their assistance with data collection.

\section{Authors' Contributions}

S.D.S. and J.K. designed the experiment. S.D.S., J.K., and B.K.F. conducted the experiment, analyzed the data, and wrote the article.

\section{Author Disclosure Statement}

No competing financial interests exist.

\section{References}

Abou-Elseoud A, Starck T, Remes J, Nikkinen J, Tervonen O, Kiviniemi V. 2010. ICA Model order selection of task coactivation networks. Hum Brain Mapp 31:1207-1216.

Andrews-Hanna JR. 2012. The brain's default network and its adaptive role in internal mentation. Neuroscientist 18:251-270.

Baron-Cohen S, Wyke MA, Binnie C. 1987. Hearing words and seeing colours: an experimental investigation of a case of synaesthesia. Perception 16:761-767.

Barratt EL, Davis NJ. 2015. Autonomous Sensory Meridian Response (ASMR): a flow-like mental state (No. e719v1). PeerJ 3:e851.

Beckmann CF, DeLuca M, Devlin JT, Smith SM. 2005. Investigations into resting-state connectivity using independent component analysis. Philos Trans R Soc London B Biol Sci 360:1001-1013.

Benedek M, Kaernbach C. 2011. Physiological correlates and emotional specificity of human piloerection. Biol Psychol 86:320-329.

Bilevicius E, Smith SD, Kornelsen J. 2018. Resting state network functional connectivity patterns associated with the mindful attention awareness scale. Brain Connect 8:1.

Biswal B, Yetkin FZ, Haughton VM, Hyde JS. 1995. Functional connectivity in the motor cortex of resting human brain using echo-planar MRI. Magn Reson Med 34:537-541.

Broyd S, Demanuele C, Debener S, Helps S, James C, SonguaBarke E. 2009. Default-mode brain dysfunction in mental disorders: a systematic review. Neurosci Biobehav Rev 33:279-296.

Buckner RL, Andrews-Hanna JR, Schacter DL. 2008. The brain's default network: anatomy, function, and relevance to disease. Ann N Y Acad Sci 1124:1-38.

Chen Y, Meng X, Hu Q, Cui H, Ding Y, Kang L, et al. 2016. Altered resting-state functional organization within the central executive network in obsessive-compulsive disorder. Psychiatry Clin Neurosci 70:448-456.

Craig AD. 2009. How do you feel-now? The anterior insula and human awareness. Nat Rev Neurosci 10:59-70.

Critchley HD, Tang J, Glaser D, Butterworth B, Dolan RJ. 2005. Anterior cingulate activity during error and autonomic response. Neuroimage 27:885-895.

Critchley HD, Wiens S, Rotshtein P, Öhman A, Dolan RJ. 2004. Neural systems supporting interoceptive awareness. Nat Neurosci 7:189-195. 
Cytowic RE. 1993. The Man Who Tasted Shapes. New York: G. P. Putnam's Sons.

Damoiseaux JS, Rombouts SA, Barkhof F, Scheltens P, Stam CJ, et al. 2006. Consistent resting-state networks across healthy subjects. Proc Natl Acad Sci U S A 103:13848-13853.

del Campo MA, Kehle TJ. 2016. Autonomous sensory meridian response (ASMR) and frisson: mindfully induced sensory phenomena that promote happiness. Int J School Educ Psychol 4:99-105.

De Luca M, Beckmann CF, De Stefano N, Matthews PM, Smith SM. 2006. fMRI resting state networks define distinct modes of long-distance interactions in the human brain. Neuroimage 29:1359-1367.

Doll A, Hölzel BK, Boucard CC, Wohlschlager AM, Sorg C. 2015. Mindfulness is associated with intrinsic functional connectivity between default mode and salience networks. Front Hum Neurosci 9:461.

Dovern A, Fink GR, Fromme CB, Wohlschläger AM, Weiss PH, Riedl V. 2012. Intrinsic network connectivity reflects consistency of synesthetic experiences. J Neurosci 32:76147621.

Esposito F, Scarabino T, Hyvärinen A, Himberg J, Formisano E, Comani S, et al. 2005. Independent component analysis of fMRI group studies by self-organizing clustering. Neuroimage 25:193-205.

Fredborg BK, Clark J, Smith SD. 2017. The big five personality traits and Autonomous Sensory Meridian Response (ASMR). Front Psychol 8:247.

Friston KJ. 2011. Functional and effective connectivity: a review. Brain Connect 1:13-36.

Gagnepain P, Hulbert J, Anderson MC. 2017. Parallel regulation of memory and emotion supports the suppression of intrusive memories. J Neurosci 37:6423-6441.

Grabenhorst F, Rolls ET. 2011. Value, pleasure, and choice in the ventral prefrontal cortex. Trends Cogn Sci 15:56-67.

Greicius MD, Srivastava G, Reiss AL, Menon V. 2004. Defaultmode network activity distinguishes Alzheimer's disease from healthy aging: evidence from functional MRI. Proc Natl Acad Sci U S A 101:4637-4642.

Gusnard D, Raichle ME. 2001. Searching for a baseline: functional imaging and the resting human brain. Nat Rev Neurosci 2:685-694.

Harrison L, Loui P. 2014. Thrills, chills, frissons, and skin orgasms: toward an integrative model of transcendent psychophysiological experiences in music." Front Psychol 5:790.

Hickok G, Poeppel D. 2007. The cortical organization of speech processing. Nat Rev Neurosci 8:393-402.

Hyvärinen A, Oja E. 2000. Independent component analysis: algorithms and applications. Neural Netw 13:411-430.

Joel SE, Caffo BS, Zijl PCMV, Pekar JJ. 2011. On the relationship between seed-based and ICA-based measures of functional connectivity. Magn Reson Med 66:644-657.

Lochte, BC, Guillory, SA, Richard, CAH, Kelley, WM. 2018. An fMRI investigation of the neural correlates underlying the autonomous sensory meridian response (ASMR). Bioimpacts 8:295-304.

McCrae, RR. 2007. Aesthetic chills as a universal marker of openness to experience. Motiv Emot 31:5-11.

Menon V. 2015. Salience network. In: Toga AW (ed.) Brain Mapping: An Encyclopedic Reference, vol. 2. Boston: Academic Press, pp. 597-611.

Mikl M, Marecek R, Hlustík P, Pavlicová M, Drastich A, Chelbus $\mathrm{P}$, et al. 2008. Effects of spatial smoothing on fMRI group inferences. Magn Reson Imaging 26:490-503.
Morelli SA, Sacchet MD, Zaki J. 2015. Common and distinct neural correlates of personal and vicarious reward: a quantitative meta-analysis. Neuroimage 112:244-253.

Pereira MG, Oliveira L, Erthal FS, Joffily M, Mocaiber IF, Volchan E, Pessoa L. 2010. Emotion affects action: midcingulate cortex as a pivotal node of interaction between negative emotion and motor signals. Cogn Affect Behav Neurosci 10:94-106.

Poerio GL, Blakey E, Hostler TJ, Veltri T. 2018. More than a feeling: Autonomous sensory meridian response (ASMR) is characterized by reliable changes in affect and physiology. PLoS ONE 13:e0196645.

Raichle ME. 2015. The brain's default mode network. Ann Rev Neurosci 38:433-447.

Raichle ME, MacLeod AM, Snyder AZ, Powers WJ, Gusnard DA, Shulman GL. 2001. A default mode of brain function. Proc Natl Acad Sci U S A 98:676-682.

Ramachandran VS, Hubbard EM. 2003. The phenomenology of synaesthesia. J Consciousness Stud 10:49-57.

Ray KL, McKay DR, Fox PM, Riedel MC, Uecker AM, Beckmann CF, et al. 2013. ICA model order selection of task co-activation networks. Front Neurosci 7:237.

Rosazza C, Minati L. 2011. Resting-state brain networks: literature review and clinical applications. Neurol Sci 32:773-785.

Ruehl RM, Hinkel C, Bauermann T, zu Eulenburg P. 2017. Delineating function and connectivity of optokinetic hubs in the cerebellum and the brainstem. Brain Struct Funct 222:4163-4185.

Seeley WW, Menon V, Schatzberg AF, Keller J, Glover GH, Kenna H, et al. 2007. Dissociable intrinsic connectivity networks for salience processing and executive control. J Neurosci 27:2349-2356.

Sestieri C, Corbetta M, Romani GL, Shulman GL. 2011. Episodic memory retrieval, parietal cortex, and the default mode network: functional and topographic analyses. J Neurosci 31:4407-4420.

Smith SD, Fredborg BK, Kornelsen J. 2017. An examination of the default mode network in individuals with Autonomous Sensory Meridian Response (ASMR). Soc Neurosci 12:361-365.

Spreng RN, Mar RA, Kim AS. 2009. The common neural basis of autobiographical memory, prospection, navigation, theory of mind, and the default mode: a quantitative meta-analysis. $\mathrm{J}$ Cogn Neurosci 21:489-510.

Sridharan D, Levitin DJ, Menon V. 2008. A critical role for the right fronto-insular cortex in switching between centralexecutive and default-mode networks. Proc Natl Acad Sci U S A 105:2569-12574.

Ward J. 2013. Synesthesia. Ann Rev Psychol 64:49-75.

Williams PG, Johnson KT, Curtis BJ, King JB. 2018. Individual differences in aesthetic engagement are reflected in restingstate fMRI connectivity: implications for stress resilience. Neuroimage 179:156-165.

Woo CW, Krishnan A, Wager TD. 2014. Cluster-extent based thresholding in fMRI analyses: pitfalls and recommendations. Neuroimage 91:412-419.

Address correspondence to: Stephen D. Smith

Department of Psychology

University of Winnipeg 515 Portage Avenue Winnipeg R3B $2 E 9$

Canada

E-mail: s.smith@uwinnipeg.ca 